\title{
DRIVER PREFERENCE OF COLLISION WARNING STRATEGY AND MODALITY
}

\author{
Joshua D. Hoffman, John D. Lee \\ Center for Computer Aided Design \\ Department of Mechanical and Industrial Engineering \\ University of Iowa \\ Iowa City, IA \\ E-mail: jdlee@engineering.uiowa.edu \\ Elizabeth M. Hayes \\ DAIMLERCHRYSLER Research and Technology \\ Portland, OR
}

\begin{abstract}
The success of collision warning systems depends on how well the algorithm and driver interface are tailored to driver capabilities and preferences. An effective collision warning system must promote a timely and appropriate driver response while minimizing annoyance associated with nuisance warnings. A within-subject experimental design examined warning strategy and modality by contrasting graded and imminent warning strategies with auditory and haptic warning modalities. Presented on a high, head-down display placed directly in front of the driver, visual warnings were displayed in the form of graded bars representing severity, or by an imminent collision icon. Visual warnings were paired with either an auditory warning or a haptic warning in the form of a vibrating seat. Results suggest that haptic warnings may be preferred over auditory warnings, with graded haptic warnings being preferred more than imminent haptic warnings. These results support previous findings of greater acceptance of graded compared to imminent warnings, and no decrement in performance or acceptance of a haptic versus an auditory warning.
\end{abstract}

